\title{
Distribution of motor unit potential velocities in short static and prolonged dynamic contractions at low forces: use of the within-subject's skewness and standard deviation variables
}

\author{
E. G. Klaver-Król · N. R. Henriquez • S. J. Oosterloo • \\ P. Klaver · J. M. Bos • M. J. Zwarts
}

Accepted: 14 May 2007 / Published online: 15 September 2007

(C) Springer-Verlag 2007

\begin{abstract}
Behaviour of motor unit potential (MUP) velocities in relation to (low) force and duration was investigated in biceps brachii muscle using a surface electrode array. Short static tests of $3.8 \mathrm{~s}$ (41 subjects) and prolonged dynamic tests (prolonged tests) of 4 min (30 subjects) were performed as position tasks, applying forces up to $20 \%$ of maximal voluntary contraction (MVC). Four variables, derived from the inter-peak latency technique, were used to describe changes in the surface electromyography signal: the mean muscle fibre conduction velocity (CV), the proportion between slow and fast MUPs expressed as the within-subject skewness of MUP velocities, the within-subject
\end{abstract}

\section{E. G. Klaver-Król · N. R. Henriquez}

Department of Neurology and Clinical Neurophysiology,

Hospital Group Twente, Geerdinksweg 141,

Hengelo, The Netherlands

\section{S. J. Oosterloo}

Faculty of Behavioral Sciences, University of Twente,

Enschede, The Netherlands

P. Klaver

Department of Psychology, Cognitive Neuroscience

and Children's University Hospital,

University of Zurich, Zurich, Switzerland

J. M. Bos

Faculty of Business, Public Administration and Technology,

University of Twente, Enschede, The Netherlands

\section{J. Zwarts}

Department of Clinical Neurophysiology, Institute of Neurology,

University Medical Centre, Nijmegen, The Netherlands

E. G. Klaver-Król ( $\square)$

PO Box 546, 7550 AM Hengelo, The Netherlands

e-mail: eklaver@planet.nl standard deviation of MUP velocities [SD-peak velocity $(\mathrm{PV})]$, and the amount of MUPs per second (peak frequency $=\mathrm{PF}$ ). In short static tests and the initial phase of prolonged tests, larger forces induced an increase of the CV and PF, accompanied with the shift of MUP velocities towards higher values, whereas the SD-PV did not change. During the first 1.5-2 min of the prolonged lower force levels tests (unloaded, and loaded 5 and 10\% MVC) the CV and SD-PV slightly decreased and the MUP velocities shifted towards lower values; then the three variables stabilized. The PF values did not change in these tests. However, during the prolonged higher force (20\% MVC) test, the $\mathrm{CV}$ decreased and MUP velocities shifted towards lower values without stabilization, while the SD-PV broadened and the PF decreased progressively. It is argued that these combined results reflect changes in both neural regulatory strategies and muscle membrane state.

Keywords Motor unit potential - Conduction velocity distribution $\cdot$ Surface electromyography $\cdot$ Dynamic contraction $\cdot$ Position task $\cdot$ Skewness

$\begin{array}{ll}\text { Abbreviations } \\ \text { MU } & \text { Motor unit } \\ \text { MUP } & \text { Motor unit potential } \\ \text { SEMG } & \text { Surface electro-myography } \\ \text { MVC } & \text { Maximal voluntary contraction } \\ \text { BB } & \text { Biceps brachii muscle } \\ \text { IPL } & \text { Inter-peak latency method } \\ \text { CV } & \text { Mean muscle fibre conduction velocity } \\ \text { PV } & \text { Peak velocity = MUP propagation velocity } \\ \text { Sk-PV } & \text { Skewness of the within-subject PVs } \\ \text { SD-PV } & \text { Standard deviation of the within-subject PVs } \\ \text { PF } & \text { Peak frequency = MUP frequency = amount of } \\ & \text { MUPs per second }\end{array}$




\section{Introduction}

Diverse laboratory conditions have been used in surface electromyography (sEMG) studies in order to gain insights into the neural regulatory strategies and muscle membrane alterations. The influence of force load on sEMG can be investigated by using force tasks or position tasks. The majority of studies have been performed as force tasks, which means that the subject controls the effort by maintaining a target force while the limb position is fixed. During the position tasks, in contrast, an inertial load is applied while the subject controls a target limb position. Both force and position tasks can be performed in static or dynamic conditions. Examples of the static force tasks are the well-known isometric experiments, with higher and lower force levels. In the recent past, force tasks in dynamic conditions have been performed sporadically, such as the cycling experiments by Pozzo et al. (2004) and Farina et al. (2004). Since Hunter et al. (2002) found that, with the same load torque, position tasks resulted in a shorter endurance time than force tasks, suggesting different regulatory mechanism for the both type of tasks, the position task studies gained field. Most position task experiments have been performed in static conditions, evaluating the underlying physiological phenomena during force versus position tasks (Hunter et al. 2002, 2003; Hunter and Enoka 2003; Rudroff et al. 2005, 2007). Potvin (1997) has described the changes in the sEMG during position tasks in dynamic conditions. Previous findings suggest that, as compared with force tasks, position tasks induce greater synaptic input into the motor neurons (Mottram et al. 2005a) and greater adaptation in the motor unit discharge (MacGillis et al. 2003).

Changes in muscle activity during (static and dynamic) position tasks have been assessed using two of the three traditional sEMG parameters, the power spectrum and the global sEMG amplitude. However, the third parameter, the mean muscle fibre conduction velocity (CV), has been lacking. Spectral estimates are generally accepted in fatigue experiments as equivalents of CV because they highly correlate with the CV's changes (Bigland-Ritchie 1981; Eberstein and Beatie 1985; Arendt-Nielsen and Mills 1985). But this correlation holds true only for the constant forces and isometric conditions; thus, a replacement of $\mathrm{CV}$ by power spectrum assessments does not always seem feasible (Farina et al. 2002; Broman et al. 1985). The favour of CV above power spectrum is, furthermore, that it renders direct and absolute values of conduction velocity, and is less sensitive to the anatomical local relationships, such as depth of the motor unit (MU) in relation to the muscle and skin surface (Farina et al. 2002). Yet the limitation of these global $\mathrm{CV}$ measurements remains the lack of sensitivity to the changes at the level of an individual motor unit potential/ motor unit.
To accommodate with these limitations of $\mathrm{CV}$, researchers have recently been trying to disentangle the propagation velocities of individual motor unit potentials (MUPs) from sEMG. One of the methods is the inter-peak latency method (IPL) proposed by Lange et al. (2002). The principle comprises calculating conduction velocities of the MUPs from the latencies between paired MUPs of two differential sEMG signals obtained parallel to the muscle fibres, and the distance between the recording electrodes. The negative peaks of two paired MUPs are then the elements determining the interpeak latency. As motor unit propagation velocity reflects the intrinsic physiological properties of a MU, such as fast-twitch or slow-twitch type (Buchthal et al. 1973; Andreassen and Arendt-Nielsen 1987), the IPL method renders many diverse MUP velocities. Lange at al. (2002) proposed using the standard deviation of MUP velocities as an additional measure that offers information about muscle fibre properties. Changes in these velocities during prolonged effort may indicate, for example, slowing/fatigue of the activated motor units and/or appearance of fast/newly recruited motor units. Such shifts in the activated MUs' populations were shown by Houtman et al. (2003) by eliciting the MUP velocities with the IPL method and presenting their distribution in histograms. The IPL method has not been applied much. It yields insights into the diversity of MUP velocities and thereby the underlying changes in the MU activity. The method is simple and does not require expensive apparatus or software. When compared with techniques that assess the propagation patterns of MUPs by multi-channel/spatial resolution sEMG (Masuda and Sadoyama 1986; Rau et al. 1997), the IPL method is unable to distinguish and follow individual MUPs belonging to the specific MUs.

In the present study, the sEMG signal was described with four parameters derived from the IPL method: (1) the mean muscle $\mathrm{CV}$ which was the average of the obtained MUP velocities; the two statistical distribution variables, which were: (2) the within-subject MUP velocities' skewness [Sk-peak velocity (PV)] and (3) the within-subject MUP velocities' standard deviation (SD-PV), and (4) the peak frequency $(\mathrm{PF})$, a variable expressing the amount of MUP activity (number of peaks = MUPs) per second.

The aim of the present study was to investigate with the four parameters the changes in MUPs' velocities of the biceps brachii muscle (BB) during prolonged dynamic position tasks, in dependence of (low) force and duration. It is chosen for the dynamic position tasks as study design because their physiology promised the finding of a large variety of MUP velocities (great amount of activity due to the position tasks character, and diversity because of the recruitment/derecruitment changes within the dynamic cycle). Whole cycles of movement with their concentric and eccentric phases were analyzed together in order to evaluate 
a total of the sEMG activity with its evolution over time. To highlight the initial changes with the effect of force on it, the changes during the first $14.4 \mathrm{~s}$ of the dynamic tasks were evaluated separately. Additively, short static position tasks were performed in order to show the (early) changes on force, without any influence of movements on the signal.

\section{Methods}

\section{Subjects}

The study involved short static and prolonged dynamic experiments. Forty-one healthy and physically active males $(24.8 \pm 6.7$ years, from 17 to 48$)$ (mean \pm SD) volunteered for the first experiment and 30 randomly chosen subjects from that group (25.4 \pm 7.6 years, from 18 to 42$)$ participated in both experiments. Exclusion criteria were drug abuse and the practice of bodybuilding. Three from a total of 44 subjects were excluded because of the impossibility to obtain a required correlation coefficient between the sEMG signals used to estimate the parameters' values. The experimental protocol was conducted according to the Helsinki Declaration and approved by the local ethics committee. All participants gave written informed consent.

\section{Experimental set up}

Maximal voluntary contraction (MVC) of elbow flexors was measured at least 5 days before the experiment with a hand-held dynamometer (Lameris Instruments, Utrecht, The Netherlands). During the MVC measurements subjects were sitting upright. The shoulder was slightly abducted and flexed at $45^{\circ}$, the elbow was firmly sustained and flexed at $90^{\circ}$, and the forearm was supinated. The dynamometer was applied to the wrist by the break method (van der Ploeg and Oosterhuis 1991). The peak hold was switched off and the force was kept for at least $3 \mathrm{~s}$. The mean of three maximal values was taken as a MVC. The MVC was assessed with elbow at $90^{\circ}$, although the tests were performed at the elbow angle of $135^{\circ}$ (Philippou et al. 2004).

During the experiment subjects were sitting in a chair. The upper arm was slightly abducted and comfortably supported at $45^{\circ}$ of the shoulder flexion, the forearm was free. When the elbow was stretched, the line of the upper armforearm was at $45^{\circ}$ in relation to horizontal. When the elbow was flexed to the angle of $135^{\circ}$, the forearm was horizontal. The forearm was supinated during static and dynamic tests.

\section{Static tests}

Subjects were asked to hold the forearm horizontally (elbow angle was then $135^{\circ}$ ). A visual bar helped to maintain the correct (horizontal) position of the forearm. In the loaded tests a sack filled with lead and sand was placed in the palm. Three levels of force were applied in blocks that were 3 min apart: unloaded, loaded 10 and 20\% MVC. A block consisted of three tests (three repetitions at the same level of force); every test lasted for $3.8 \mathrm{~s}$ and was within a block separated $30 \mathrm{~s}$ from one another.

\section{Dynamic tests}

All participants of the dynamic tests underwent previously static tests, separated by $5 \mathrm{~min}$. Subjects were asked to swing the forearm from the stretched (elbow angle $180^{\circ}$ ) to horizontal position (elbow angle $135^{\circ}$ ), thus moving over an angle of $45^{\circ}$. They did it within a rate of 40 beats per minute (one up-and-down movement in one beat), given by a metronome sound. The visual bar indicated the horizontal position to which the lower arm returned after being stretched. Four force levels were applied: unloaded, and loaded 5, 10 and 20\% MVC. The tests lasted for $4 \mathrm{~min}$ and were separated by $5 \mathrm{~min}$.

\section{EMG recording}

Measurements were performed on the short head of the BB of a dominant arm. A surface electrode array consisted of three golden-coated electrodes (Harwin, P25-3526), diameter $1.5 \mathrm{~mm}$, insulated in synthetic material plate, with a $10 \mathrm{~mm}$ distance between the electrodes (Sadoyama et al. 1985). The skin was cleaned with $95 \%$ ethanol. The electrode array was placed parallel to the muscle fibres (Sollie et al. 1985). The proximal electrode was positioned exactly on the distal one-quarter point of the upper arm, measured between the coracoid and the elbow crease. This place of the electrode was about halfway between the endplate zone and the tendon, securing a sufficient distance from the endplate (Sadoyama et al. 1985; Masuda and Sadoyama 1987). Bipolar derivation was made from the proximal to distal direction. The optimal electrode position was controlled by both the observation of the signal on the monitor and the estimation of a correlation coefficient (CC) of the two sEMG signals, which was accepted at $r>0.7$ for unloaded, $r>0.85$ for $5 \% \mathrm{MVC}$ and $r>0.9$ for higher loaded tests. (During the static and dynamic tests, the maximal CC for unloaded arm was usually lower than that for loaded arm, which was consistent with Hogrel et al. 1998, who have estimated for an unloaded arm $r>0.7)$. The ground electrode was placed on the lateral upper arm, slightly proximal from the derivation electrode. The temperature sensor was medial on the upper arm. Two obtained signals were differentially amplified (gain 2,000-10,000×) and band pass filtered at $2-250 \mathrm{~Hz}$ by EMG apparatus (Viking IV, US). 


\section{Data processing}

The signals were simultaneously A/D converted (sampling $10 \mathrm{kHz}, 12$ bits acquisition). Data were stored on a personal computer. The signal was analyzed with LabVIEW 6.1 that also facilitated a partial on-line analysis. The peak selection and the correlation coefficient assessments for both static and dynamic tests were performed on $0.2 \mathrm{~s}$ epochs. In the static tests, measurements were taken every second during $0.8 \mathrm{~s}$ (comprising 4 epochs of $0.2 \mathrm{~s}$ ). A static test was of $3.8 \mathrm{~s}$ duration and was repeated three times for each force level. The statistical analyses were performed on the data of these three repeated tests taken together. In the dynamic tests, the data were assembled every $30 \mathrm{~s}$ during $14.4 \mathrm{~s}$ (comprising 72 epochs of $0.2 \mathrm{~s}$ ). The test duration was 4 min.

\section{Peak selection}

The basic principle was that of Lange et al. (2002). The software was custom designed and written in LabView. The orientation of the signals is up-negative. 1st step: finding zero line. 2nd step: finding the peak-to-peak amplitude of the largest MUP in an epoch of $0.2 \mathrm{~s} .3 \mathrm{~d}$ step: finding a peak-decline structure. The peak-decline is defined as a structure with a decline of $\geq 20 \%$ compared with the largest MUP amplitude of a 0.2 epoch, over $\leq 4 \mathrm{~ms}$ ( $\leq 40$ sample points). $3 \mathrm{~d}$ step: finding a peak. A peak is the highest (most negative) point previous to the decline, and must be $\geq 10 \mu \mathrm{V}$ (the threshold of the noise level). 4th step: finding a pair peak. A pair peak is a peak in the second signal with the properties as previous, found in a time window between 1.49 and $4 \mathrm{~ms}$ after the peak of the first signal. This window is chosen assuming the physiological CV values of $2.5-6.67 \mathrm{~m} / \mathrm{s}$. 5th step: excluding double peak. If a peak from the first signal matches two different peaks from the second signal, then the first peak from the second signal is true. If during the fatiguing tests the CV severely diminishes, then the low limit of velocity is put at $1.3 \mathrm{~m} / \mathrm{s}$, making a window of $1.49-7.68 \mathrm{~ms}$. As an objective pragmatic criterion to this change a lowering of the peak frequency with $\leq 30 \%$ was assumed, as compared with the first value of the $20 \%$ MVC test.

\section{The parameters}

The following calculations were performed: (1) basic calculation of PV, following the IPL method; (2) mean CV, expressed as an average value of the PVs; (3) within-subject skewness of the peak velocities (Sk-PV), expressed as a skewness of a PVs' population of a subject; (4) withinsubject standard deviation of the peak velocities (SD-PV), expressed as a standard deviation of a PVs' population of a subject; and (5) peak frequency (PF), expressed as a number of peaks per second.

\section{Statistics}

One-way analysis of variance (ANOVA) with repeated measures on force was used to compare the dependent variables for static tests (three levels) and for the initial values of the dynamic tests (four levels). A two-way ANOVA with repeated measures on force ( four levels) and time (nine levels) was used to compare variables during the dynamic tests. In the case of interactions between force and time, to describe changes over time, when appropriate, four separated ANOVAs were performed with smaller time windows of $0-60,60-120,120-180$ and $180-240$ s. To be assured that the dependent variables met parametric assumptions, plots of residues were produced with SPSS program, model control as suggested by Kutner et al. (2005, p. 1157). No relevant deviations of model were detected. Pearson correlation coefficients were calculated to evaluate associations between variables. A level of $P<0.05$ was used to identify statistical significance.

\section{Results}

Subjects' physical characteristics are presented in the Table 1. The force of the biceps correlated positively with the upper arm circumference $(r=0.484, P<0.01)$. No association was found between either force or upper arm circumference and the sEMG variables. The average skin temperature increased during the dynamic tests by $1.65^{\circ} \mathrm{C}$ $(P<0.001)$; it did not change during the static tests.

\section{Static tests}

\section{Mean muscle fibre conduction velocity $(\mathrm{CV})$}

Histograms in Fig. 1 show an example of a PVs' population in one subject during the static tests at three levels of force: unloaded, 10 and 20\% MVC. Figure 2a presents the averages of the CVs over 41 subjects, calculated from the subject's PVs. The CV of the unloaded test was the lowest $\left(3.92 \pm \mathrm{SD} 0.27 \mathrm{~m} \mathrm{~s}^{-1}\right)$ and it increased with augmenting force levels (effect of force $P<0.001$ ). A positive correlation existed between the CVs of unloaded test with $10 \%$ MVC, and 10\% with 20\% MVC (all $r>0.505, P<0.05$ ).

\section{Skewness of peak velocities (Sk-PV)}

Figure $2 b$ presents the averages of the within-subject PVs' skewnesses over 41 subjects (see also the histograms of PVs in Fig. 1). In all the tests a moderate positive 
Table 1 Characteristics of 41 participants to the static tests; 30 of them participated in the dynamic tests as well a From the coracoid to the elbow crease

b From the lateral epicondyle to the wrist crease

c From the lateral epicondyle to the middle of the palm

\begin{tabular}{lcccrr}
\hline & $N$ & Minimum & Maximum & Mean & SD \\
\hline Age (years) & 41 & 16 & 48 & 24.7 & 6.7 \\
Height (cm) & 41 & 166.0 & 197.0 & 183.2 & 8.0 \\
Weight (kg) & 41 & 60.0 & 95.0 & 74.3 & 9.2 \\
Force (N) right & 38 & 148.5 & 346.5 & 246.1 & 41.9 \\
Force (N) left (for left-handed) & 3 & 262.3 & 267.3 & 265.1 & 2.5 \\
Skin thickness (mm) & 41 & 1.4 & 4.2 & 2.4 & .7 \\
Circumference upper arm $(\mathrm{cm})$ & 41 & 24.0 & 31.0 & 27.5 & 2.0 \\
Length of biceps $(\mathrm{cm})^{\mathrm{a}}$ & 41 & 30.50 & 39.50 & 35.4 & 2.0 \\
Length lower arm/radius $(\mathrm{cm})^{\mathrm{b}}$ & 33 & 24.0 & 30.0 & 26.4 & 1.6 \\
Length lower arm/palm $(\mathrm{cm})^{\mathrm{c}}$ & 33 & 30.0 & 36.0 & 33.1 & 1.6 \\
Initial skin temp. $\left({ }^{\circ} \mathrm{C}\right)$ & 41 & 30.8 & 33.8 & 32.3 & .8 \\
Temp. upper arm before dyn. tests $\left({ }^{\circ} \mathrm{C}\right)$ & 30 & 30.8 & 34.2 & 32.3 & .9 \\
Temp. upper arm after dyn. tests $\left({ }^{\circ} \mathrm{C}\right)$ & 30 & 32.2 & 35.7 & 33.9 & 1.1 \\
Room temp. $\left({ }^{\circ} \mathrm{C}\right)$ & 41 & 20.0 & 24.0 & 22.3 & 1.0 \\
\hline
\end{tabular}

\section{Peak frequency $(P F)$}

Figure $2 \mathrm{~d}$ shows the averages of PF in the static tests. The $\mathrm{PF}$ increased with increasing forces (effect of force $P<0.001)$.

\section{Dynamic tests}

\section{Muscle fibre conduction velocity $(\mathrm{CV})$}

Histograms in Fig. 3 show the PVs estimated from one subject during dynamic tests at four levels of force: unloaded, loaded with 5, 10 and 20 MVC. Figure 4a shows the averages of CV calculated from the subject's PVs over 30 subjects. As in the static tests, the initial CV of the dynamic tests increased with level of force (effect of force $P<0.001$ ). Further, a positive correlation was found between the $\mathrm{CV}$ of the static tests and the initial $\mathrm{CV}$ of the respective dynamic tests (all $r>0.409, P<0.05$ ). The lowest initial $\mathrm{CV}$ was that of the unloaded test with about $4(3.6-4.5) \mathrm{m} \mathrm{s}^{-1}$ and the highest was at 20\% MVC with approximately 4.6 (4.0-5.15) $\mathrm{m} \mathrm{s}^{-1}$, increasing from the unloaded to $20 \% \mathrm{MVC}$ test with $14 \pm 9.6 \%$. During all the dynamic tests the CV significantly declined (effects of time for the unloaded test $P<0.02$; for other tests $P<0.001$ ), whereby the decline was steeper with larger forces (interaction between force and time, $P<0.001$ ). At the three lowest force levels (unloaded, and loaded 5 and 10\% MVC), the CV had two phases: a decline phase lasting for about 120-150 s and a steady phase continuing to the end of a test. The steady state values approached the level of the unloaded test. During the $20 \%$ MVC test, however, the CV continued to decline, without a stable phase. Twelve of 30 subjects $(40 \%)$ reported fatigue during the $20 \%$ MVC test and terminated the task the force levels increased (effect of force n.s.). 

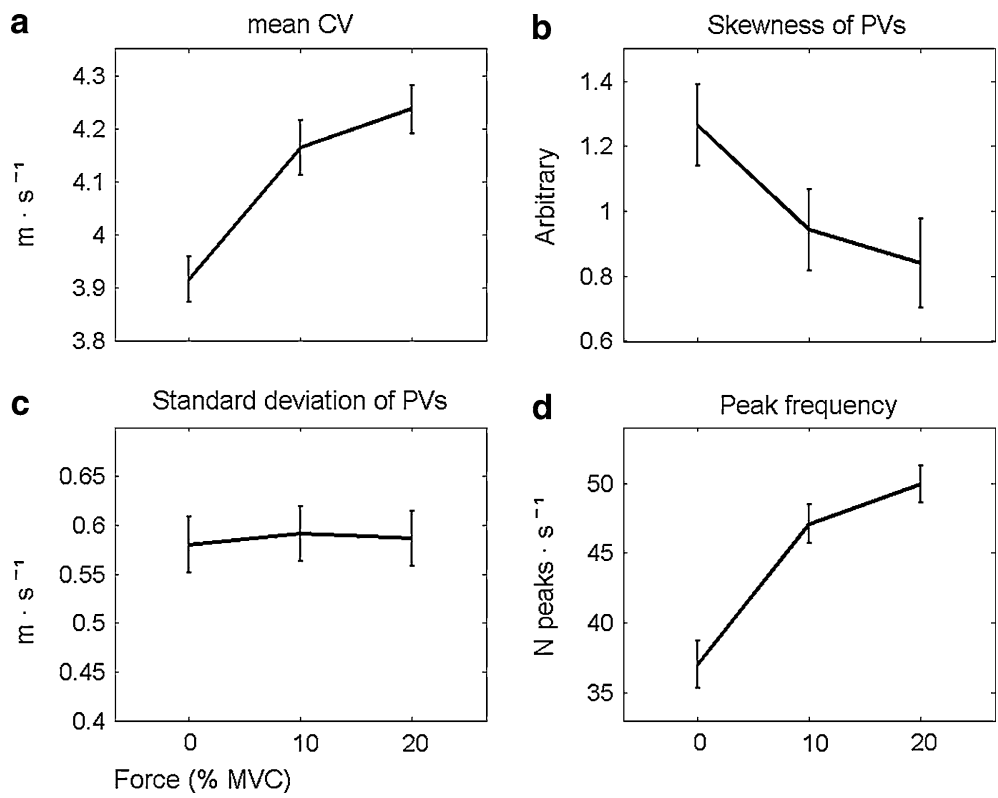

Fig. 2 Behaviour of peak velocities (PVs) as effect of force, expressed with four parameters. a Mean conduction velocity $(\mathrm{CV})$; $\mathbf{b}$ skewness of within-subject PVs; $\mathbf{c}$ SD of within-subject PVs $(S D)$; and $\mathbf{d}$ number of peaks per second (peak frequency $=\mathrm{PF}$ ). Averages and standard errors are given, obtained from 41 subjects in short static tests at three levels

of force: unloaded, and loaded 10 and $20 \%$ of maximal voluntary contraction. With increasing forces, the $\mathrm{CV}$ and amount of activity $(P F)$ increases, accompanied with augmenting proportion of fast peaks (the skewness value diminishes). However, the spread of peak velocities within an individual $(S D)$ does not change
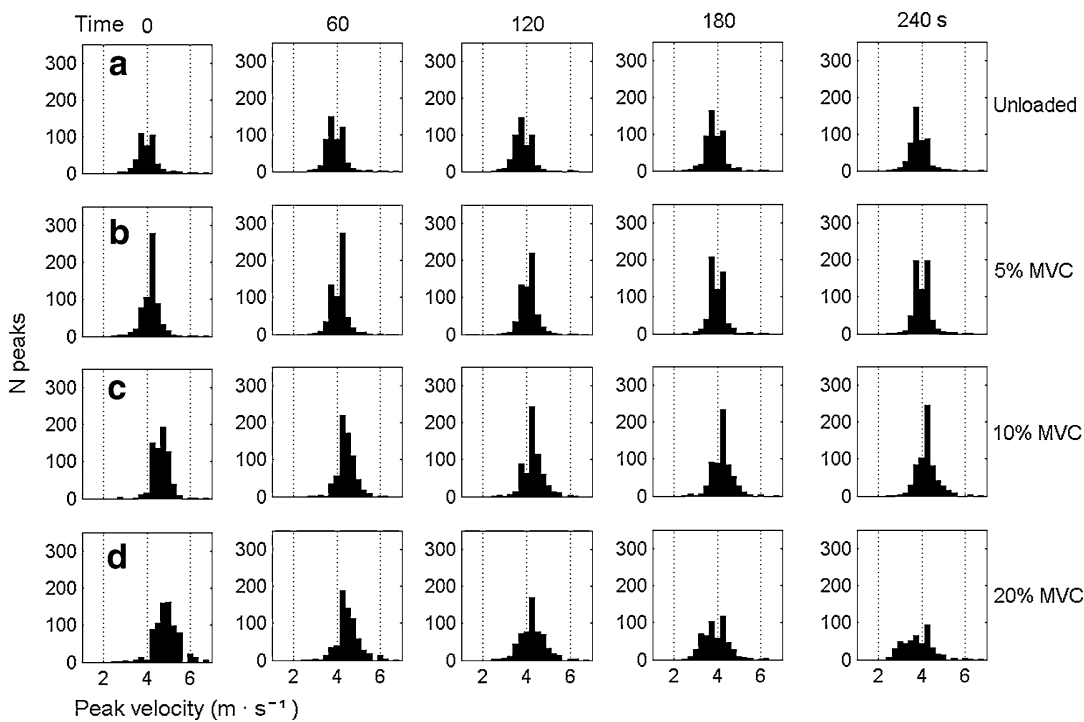

Fig. 3 Changes in the distribution of peak velocities $(P V s)$ over time for different levels of force. The PVs are obtained from one subject (the same as in Fig. 1 for static tests) during prolonged dynamic contractions at four levels of force: unloaded, and loaded 5, 10 and $20 \%$ of maximal voluntary contraction $(M V C)$. Every histogram represents a number of PVs within a period of $14.4 \mathrm{~s}$. Initially (at time zero),

prematurely between 90 and $210 \mathrm{~s}$. The CV decreased for the fatigued subjects from $4.6 \pm 0.3(4.2-5.0)$ to $3.6 \pm 0.3$ $(3.0-4.1) \mathrm{m} \mathrm{s}^{-1}$ and for the continuing subjects from $4.5 \pm 0.3(4.0-5.15) \mathrm{m} \mathrm{s}^{-1}$ to $3.8 \pm 0.4(3.0-4.7) \mathrm{m} \mathrm{s}^{-1}$. Neither the absolute initial CV nor the end CV differed a global shift of peak velocities is visible towards higher values when forces augment. During the unloaded, and loaded 5 and 10\% MVC tests, slower peaks are moderately increasing and faster peaks are diminishing. During the $20 \%$ MVC test, the peak velocities shift considerably as a whole towards lower regions, and the amount of peaks visibly diminishes

significantly between the groups $(P=0.497$ and $P=0.124$, respectively). However, the relative decline of the $\mathrm{CV}$ tended to be larger for the fatigued subjects compared with the continuing subjects, for fatigued being about $-20(-7$ to $-35) \%$ and for continuing -15 (+7 to -28$) \%$; $P=0.074$. 

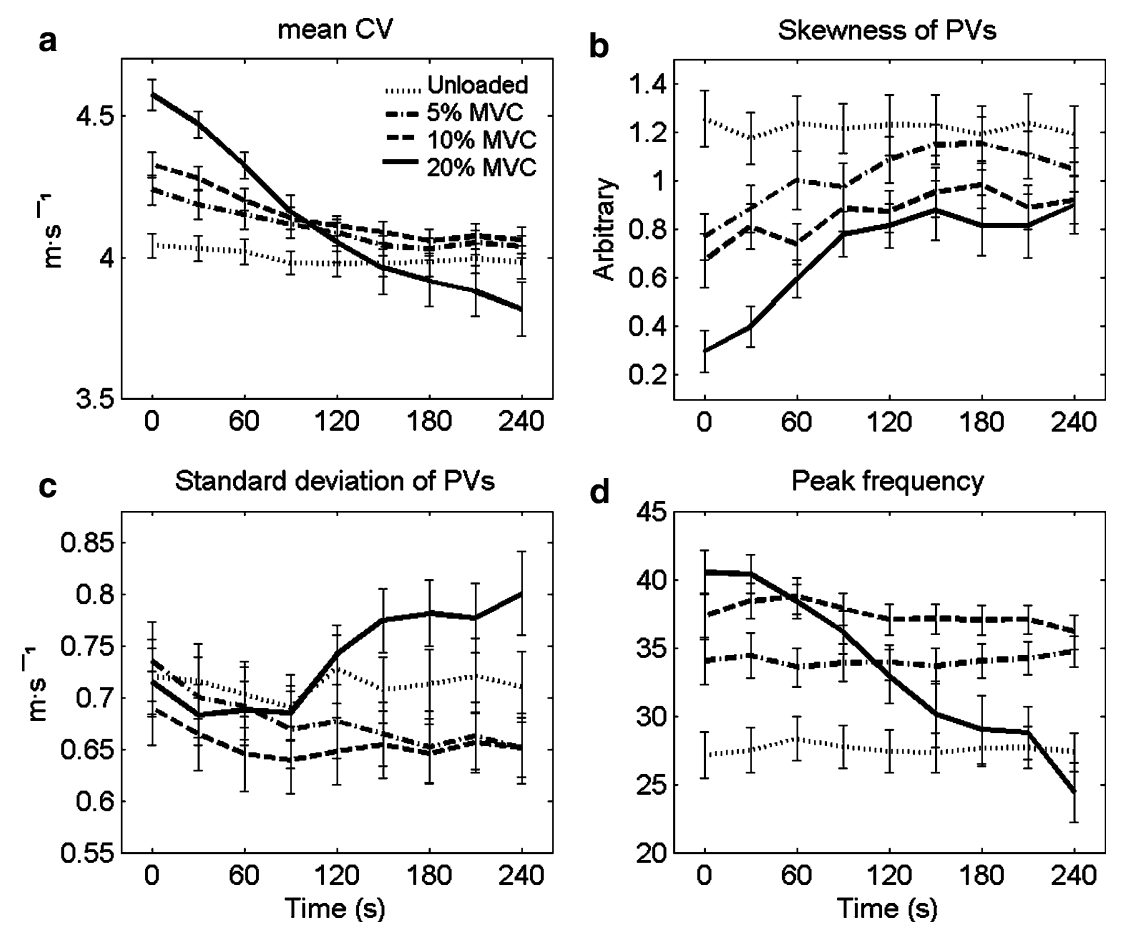

Fig. 4 Effects of force and time on the behaviour of peak velocities $(P V s)$, expressed with four parameters. a Mean conduction velocity $(C V)$; b skewness of within-subject PVs; $\mathbf{c}$ SD of within-subject PVs $(S D)$; and $\mathbf{d}$ number of peaks per second (peak frequency $=\mathrm{PF}$ ). Averages and standard errors are given, obtained from 30 subjects during prolonged dynamic tests at four levels of load: unloaded, and loaded 5, 10 and $20 \%$ of maximal voluntary contraction $(M V C)$. Note the difference in the decline pattern of the $\mathrm{CV}$ and the PF: the mean

\section{Skewness of peak velocities (Sk-PV)}

Histograms in Fig. 3 show the distribution of PVs of one subject and Fig. $4 \mathrm{~b}$ presents the averages of the within-subject's skewnesses of the PVs over 30 subjects. In the initial phase, consistent with the static tests, the PVs were most positively skewed (=skewed in favour of lower velocities) in the unloaded test, and the skewness diminished with increasing forces (effect of force $P<0.001$ ). That means that lower PVs dominated in the unloaded test and the proportion of higher PVs increased when forces augmented. Thus, in the 20\% MVC test, the initial PVs approached a normal distribution. In addition, with increasing forces the PVs as a whole group seem to shift towards higher values, as can be seen in the histograms Fig. 3a-d at time zero. During the tests, the skewness increased again, except for the unloaded test, indicating growing proportion of lower PVs over time and decreasing amount of higher PVs (histograms Fig. 3a-c). The larger the forces the steeper increase of skewness (for all tests together: effect of time $P<0.001$; interaction between force and time $P=0.005$; effect of time in unloaded test $P=$ n.s.; for the tests 5, 10 and 20\% MVC interaction between force and time $P<0.001)$. During the

muscle conduction velocity declines over time for all levels of force, while the amount of activity remains stable for the three lower force level tests (unloaded, and loaded 5 and 10\% MVC). In the higher force level test (20\% MVC), the CV starts declining immediately, while the PF declines first gradually and later on steeply. Note the stable SD values from about $90 \mathrm{~s}$ for the three lower force tests, while the SD of the higher force test (20\% MVC) clearly increases

last 2 min of the 5 and $10 \%$ MVC tests the Sk-PV stabilized. In the $20 \%$ MVC test, however, the positivity still tended to increase up to the end of the test (over the time windows 120-180 and 180-240 s: effect of time for the 5, 10 and 20\% MVC tests, n.s.; interaction between force and time, $P=0.106$; for the 5 and $10 \%$ MVC tests the effect of time, n.s.; for $20 \% \mathrm{MVC}$ test over $120-180 \mathrm{~s}$ n.s., over $180-240 \mathrm{~s}$, $P=0.052$ ). At the end of the $20 \%$ MVC test, the whole population of PVs appeared to shift towards the lower values too, as can be seen at the last two histograms in the Fig. 3d.

Taken together, in the initial phase of activity, the proportion of fast peaks increased with increasing force. In the prolonged tests loaded up to $10 \% \mathrm{MVC}$, the proportion of fast peaks declined again over the first 2 min and then stabilized at about the level of the unloaded test. During the $20 \%$ MVC test, however, the proportion of fast peaks still tended to decline up to the end of the test, accompanied with a growing amount of slow peaks.

\section{Standard deviation of peak velocities (SD-PV)}

The averages of SD-PVs of 30 subjects are presented in Fig. 4c. The initial SD-PV was for all force levels similar 
$(P=0.65)$, which resembled the static tests. The values in the dynamic tests were significantly higher compared with those of respective static tests (paired sample $t$ test for the unloaded, 10 and 20\% MVC tests, respectively $P=0.014$; $P=0.027$ and $P=0.001$ ). During the tests the SD-PV changed significantly with time, depending on the force level (for all tests effect of time $P=0.011$, interaction between force and time $P<0.001$ ). The course of the SD-PV had two phases which were different for the three lower force levels (unloaded, 5 and 10\% MVC), compared with $20 \%$ MVC. In the three lower force levels, the SD-PV first declined over about $90 \mathrm{~s}$ and then stabilized (interaction between force and time over 0-240 s $P=$ n.s.; effect of time over the time windows $0-60 \mathrm{~s} P<0.001,60-120 \mathrm{~s}$ $P=0.075 ; 120-180$ and $180-240 \mathrm{~s}$ for both $P>0.343$ ). However, during the $20 \%$ MVC test, the decline, which lasted for approximately $60 \mathrm{~s}$, was followed by an extreme increase (effect of time over 0-240 s $P<0.001$; effect of time over $0-60 \mathrm{~s} P=0.019$; over $60-120 \mathrm{~s}$, which was in opposite direction, $P=0.019$, and $120-180$ and $180-240 \mathrm{~s}$ $P<0.05)$. This pattern of results can also be seen in the histograms Fig. 3d.

\section{Peak frequency $(P F)$}

Figure $4 \mathrm{~d}$ shows averages of PF over 30 subjects. The initial PF rose with increasing force levels (effect of force $P<0.001)$. Then, during the tests at three lowest force levels (unloaded, 5 and $10 \%$ MVC) the PF remained stable. But during the $20 \%$ MVC test the PF significantly diminished, at the beginning gradually and from about $120 \mathrm{~s}$ steeply (interaction between force and time for all the four tests, $P<0.001$; for the three lowest force levels, $P=0.234$; effect of time for the three lowest force levels $P=0.541$; effect of time for $20 \%$ MVC $P<0.001$ ). There was much variability among subjects in the size of decline in $20 \%$ MVC test. For those who were able to complete the test, the PF continued to decline up to the end, with exception of one subject in whom the PF increased instead. At 240th s the PF of the continuing subjects was reduced by $-35(-78$ to +3$) \%$.

\section{Discussion}

Changes in the distribution of MUP velocities as an effect of (low) force and duration were described with four parameters: (1) the global parameter of mean CV, (2) the within subject skewness of a population of MUP velocities; (3) the within-subject standard deviation of MUP velocities and (4) the amount of MUP activity, expressed as MUP frequency. First we will comment on the four parameters. Next, using these parameters, we will discuss the main findings.
The four parameters

The CV parameter renders a mean value of the motor unit potentials' propagation velocities. The $\mathrm{CV}$ will increase or decrease, depending on the type of the activated (fasttwitch and slow-twitch) motor units. It will also change with the alterations in muscle membrane potential, which influences the depolarisation/repolarisation processes. For example, it decreases in muscular fatigue (Stalberg 1966; Milner-Brown and Miller 1986), and increases with a smaller interstimulus interval, such as that due to the rising rate coding (Gydikov and Christova 1984; Radicheva et al. 1986; Nishizono et al. 1989). Because of the lack of studies, it is not possible to compare the CV values with those of any other position tasks experiments. However, the estimates are consistent with those of the studies using static and dynamic force tasks, especially with those of Lange et al. (2002), obtained with the inter-peak latency method.

Skewness is used as an sEMG parameter in the present study for the first time. This statistical measure of deviation from a normal distribution, in this case expresses the proportion between slower and faster MUPs within an individual. It will increase with the growing proportion of slow/ tonic/fatigue resistant MUs and will decrease with the augmenting proportion of fast/phasic/fatigable MUs. All the estimates were moderately positively skewed, which indicates a relative excess of lower MUP velocities.

The within-subject standard deviation of MUP velocities, a variable introduced by Lange et al. (2002) shows the spread of MUP velocities. For a fresh and healthy muscle, it will render information about diversity of the participating MUs. In a fatigued muscle, when membrane propagation is slowing, the SD-PV will broaden as a result of the temporal dispersion of velocities. Further, the SD-PV can be expected to narrow when the same velocities repeat, such as in a higher discharge rate of a certain group of MUs. In the brief static position tasks, the standard deviations were larger than those previously estimated by Lange and colleagues (2002) in the static force tasks, with respectively $0.55-0.62$ and $0.3-0.52 \mathrm{~m} \mathrm{~s}^{-1}$. This difference can be due to the different type of tasks, as data are available suggesting that different excitatory/inhibitory inputs to the motor neurons play a part in the position tasks and the force tasks (Rudroff et al. 2005).

In the present study, the SD-PVs of the dynamic tests were larger than those of the static tests. This difference can have different explanations. With every contraction of a dynamic cycle, the muscle fibres' diameter increases, leading to higher fibre propagation velocities (in a part of a cycle) (Arendt-Nielsen et al.1992). This problem was partially restrained by using a small movement angle of $45^{\circ}$. However, the most important role in the increase of SD-PVs' during dynamic contractions might be played by 
the cyclic changes in the motor units' discharge characteristics. Several studies deliver the supporting data. For example, during dynamic contractions the amount of activity differs between the concentric and eccentric phases, suggesting different regulatory strategies for the two phases (Potvin 1997). Previous studies have also shown that the rate of MU discharge is related to the movement's velocity, and the (angle) velocity alters depending on the elbow angle (Gillis 1972; Milner-Brown et al. 1973a; Potvin 1997). Thus, the discharge rates will alter through a cycle. In addition, eccentric movements are shown to further the activation of high-threshold (fast propagating) motor units (Komi and Tesch 1979; Nardone et al. 1989).

Peak frequency (MUP frequency) expresses the amount of MUPs in a time. To our knowledge, it is used as sEMG parameter in the present study for the first time. The PF is comparable with the zero crossing parameter (Lynn 1979; Masuda et al. 1982; Hagg 1981). It is argued that the diminishing zero crossings' number during prolonged exercises indicates decrease in MUs' activity, as a sign of fatigue (Inbar et al 1986; Hagg and Suurküla 1991). Lange et al. (2002) mentioned a number of MUPs obtained during $1.5 \mathrm{~s}$ measurements in static force tasks, which renders the frequency of about 4-5 MUPs/s for 10\% MVC test, and about $8 \mathrm{MUPs} / \mathrm{s}$ for $20 \% \mathrm{MVC}$ test. These values are much lower than ours with 37, 47 and 50 MUPs/s (for respectively unloaded, loaded 10 and 20\% MVC tests) in static position tasks. The findings are consistent with the interpretation that during position tasks more motor units are being recruited compared with force tasks, and the discharge rate of MUs is higher (Mottram et al. 2005a).

The initial changes on increasing force levels (in the static and dynamic tests)

The effects of force on the behaviour of MUPs in the short static tests and the initial phase of the prolonged dynamic tests were similar. With increasing forces the CV grew higher and MUP frequency increased. In the population of MUP velocities, not only the proportion of fast MUPs increased (see the skewness in Fig. 2b), but also the velocities as a whole shifted towards higher values (histograms in Figs. 1, 3a-d at time zero). Despite of the changes in the skewness, the standard deviation of MUP velocities remained unaltered.

The increases of the $\mathrm{CV}$ with increasing force are in accordance with the previous findings in force tasks (Naeije and Zorn 1983; Broman et al. 1985; Sadoyama and Masuda 1987; Zwarts and Arendt-Nielsen 1988; Lange et al. 2002). It is generally accepted that these increases are caused by activating high threshold/fast/phasic motor units when demands of force are augmented (Henneman et al. 1965; Milner-Brown et al. 1973b; Gantchev et al. 1992; Gazzoni et al. 2001). This explanation was supported by the increasing proportion of fast MUPs found in the present study. However, the global shift of MUP velocities towards higher values may be induced by either replacing slow MUs by fast ones, or by increasing the propagation velocity of the muscle membrane due to the rising rate coding (Radicheva et al. 1986; Nishizono et al. 1989; Van der Hoeven and Lange 1994). Little is known about changes in the withinsubject standard deviation of MUP velocities. Only Lange and colleagues (2002) mentioned (in force tasks), contrary to our results, increases between 10 and $50 \%$ MVC (and no increases between 50 and 100\%). The experiments of Lange et al. and the present short static experiments were both isometric, and the duration of the contraction did not differ much (our 3.8 vs. their $1.5 \mathrm{~s}$ ). In fact, the two studies only differed in the type of task (position tasks applied by us vs. force tasks by Lange et al.). This task difference may play a part in the discrepancy of the standard deviation, as the excitatory and inhibitory inputs for the two tasks are supposed to be different (Rudroff et al. 2005). Thus, for the two tasks different types of motor units (with different velocities) may be activated.

In short, increases of mean CV with increasing forces in the initial phase of muscle activity may be a result of both recruitment of fast/phasic motor units and faster membrane propagation.

Changes in the prolonged dynamic tests

\section{Tests loaded below 20\% MVC}

The main feature of the prolonged tests loaded 5 and $10 \%$ MVC were changes in the CV, skewness and standard deviation over the first $90-120 \mathrm{~s}$, followed by stabilizing. Thus, the CV first declined (with steeper decline for higher forces) and then stabilized at approximately the level of the unloaded test (Fig. 4a). The MUP velocities, which at the beginning of tests were shifted towards the higher values with increasing forces, re-shifted over the tests back to the lower values (skewness variable in Fig. $4 \mathrm{~b}$ and histograms in Fig. 3a-c). Subsequently, the MUP velocities stabilized nearly at the level of the unloaded test too. The standard deviation narrowed first and later on stabilized at a new level (Fig. 4c). The MUP frequency held steady over these tests at the primary level determined by the used force (Fig. 4d). We suggest that this pattern of results may reflect an emerging equilibrium between phasic and tonic MU activity.

No comparison is possible between the parameters used here and those in any other prolonged position tasks study. The decline of $\mathrm{CV}$ during contractions at low forces was in contradiction with the studies in static force tasks, which reported increases during sustained isometric contractions 
at forces of 10-25\% MVC (Zwarts and Arendt-Nielsen 1988; Arendt-Nielsen et al. 1989; Krogh-Lund and Jorgensen 1991; Krogh-Lund and Jorgensen 1992; Krogh-Lund 1993). This discrepancy may be caused by either/or both the different contraction types (static vs. dynamic) or different tasks (force vs. position tasks). Increases of the CV in prolonged isometric contractions at low force levels are supposed to be due to the recruitment of fast (anaerobic) MUs in response to the hindered blood flow (Crenshaw et al. 1997; Zwarts et al 1987). In the dynamic conditions, the blood supply is assumed to be undisturbed, so the aerobic MUs can be activated. The decline of the CV followed by stability, along with the changes in the skewness, suggest that, within the cyclically fluctuating activity, the global amount of initially recruited fast/fatigable/anaerobic MUs may successively diminish and the proportion of slow/fatigue resistant/aerobic MUs may augment. The maintaining activity of slow MUs is in accordance with the hypothesis that tonic/fatigue resistant (aerobic) MUs remain active through the whole muscular action (Grimby and Hannerz 1968; Hagg and Suurkula 1991).

On the other hand, the position character of the present tasks may have contributed to the discrepancy between the decline of $\mathrm{CV}$ in the present experiments and increases in previous studies, as firstly, the discharge characteristics of the same motor unit differ between the force and position tasks, and secondly, motor units show greater discharge adaptation during the position tasks (Mottram et al. 2005a, b; MacGilles et al. 2003). The evolution of the standard deviation parameter, with its narrowing followed by stabilizing, fits in with the idea that the rate coding may temporarily increase and consecutively adapt, resulting in a new balance.

The MUP frequency variable, expressing the amount of MU activity produced as a result of recruitment and rate coding did not change during these tests. This suggests that all the changes in recruitment and rate coding do not, in principle, affect the total amount of MU activity.

All subjects were able to complete the tests, and the sEMG parameters became stable in the course of time as well. Thus, one can assume that the three tests at lowest force levels were non-fatiguing. Taken together, the results of these apparently non-fatiguing dynamic position tasks suggest that following the initially increased activation of fast MUs, the proportion shifts after about $2 \mathrm{~min}$ in favour of slower MUs. The amount of activity seems to remain stable throughout the duration of the tests.

\section{The test at $20 \% M V C$}

The changes encountered during the prolonged test at $20 \%$ MVC differed clearly from those at lower forces (Fig. 4). During the 20\% MVC test, the CV dropped below the level of the unloaded test. At the same time the proportion of low MUP velocities increased (skewness increased), and finally the velocities' population made a global move from higher towards lower values, while their standard deviation broadened clearly. The MUP frequency, in contrast with that of non-fatiguing tests, progressively diminished (histogram in Figs. 3d, 4d).

The CV's decreases were in accordance with those shown by Farina et al. (2004) during fatiguing dynamic force tasks of vastus medialis muscle. The behaviour of the skewness and standard deviation suggests that a large majority of the MUPs became extremely slow at the end. This general slowness best matches the slowing of the muscle membrane propagation, which is generally accepted as sign of muscular fatigue (Milner-Brown et al. 1986; Miller et al. 1987). The diminishing MUP frequency can be due to the synchronisation of discharges (Datta and Stephens 1990; Semler and Nordstorm 1999) and diminishing motor unit activity (Hagg 1981; Hagg and Suurkulla 1991). The non-linear decline pattern of MUP frequency, first slow and later on steep, suggests successively exhausting available MUs' reserves, resulting in lower firing frequencies and failing recruitment. Accordingly, many subjects reported fatigue and stopped exercising.

In short, during these apparently fatiguing dynamic position tasks, a global slowing of MUP velocities appears, suggesting a fatigued muscle membrane. The amount of MU activity seems to diminish progressively and finally the recruitment stops.

Some aspects of the method must be explained. The load was applied to the palm, whereas the MVC was assessed from the wrist. By applying load to the palm, we intended to mimic natural circumstances, such as holding something in the hand. Assessment of the MVC from the palm was not feasible, however, due to the relative weakness of the wrist's flexors compared with the elbow flexors, which influenced the estimates. A reasonable alternative was to measure the MCV from the wrist. Distances measured over the forearm and the palm (Table 1) enabled calculation of the real exerted load torque, which was about $20-25 \%$ larger than the used one. The MVC was assessed at the elbow angle of $90^{\circ}$ despite performing the tests at the angle of $135^{\circ}$. The reason was that, when applying the dynamometer at the wrist with high forces, the elbow angle being at $135^{\circ}$, subjects tended to overstretch the wrist and experienced pain. This was not the case at the $90^{\circ}$ angle. The MVC values at 135 and $90^{\circ}$ were similar, which was consistent with the findings of Philippou et al. (2004), so it was chosen for assessments at $90^{\circ}$.

In conclusion, we present a set of parameters derived from the interpeak latency method, which yields information about changes in MUP velocities' distribution and amount of MUP activity. Skewness, standard deviation and 
peak frequency parameters appear to corroborate the results of a global muscle conduction velocity. Together they could contribute to quantifying the dynamics of motor unit activity and membrane fatigue. The interconnected results may be useful in ergonomics (for assessment of fatigue) and in sports (for eliciting specific capabilities, such as explosive or endurance capabilities).

Acknowledgments This study was materially supported by Hospital Group Twente Hengelo. Our thanks are extended to Staff of the Department of Neurology and Clinical Neurophysiology, Staff of the Department of Electronics, fellows-neurophysiologists of the Hospital Group Twente Hengelo, Jan Vink, Igor Klaver, Dick Stegeman, and all our subjects for their valuable contribution to this project.

\section{References}

Andreassen S, Arendt-Nielsen L (1987) Muscle fibre conduction velocity in motor units of the human anterior tibial muscle: a new size principle parameter. J Physiol 391:561-571

Arendt-Nielsen L, Mills KR (1985) The relationship between mean power frequency of the EMG spectrum and muscle fibre conduction velocity. Electroencephalogr Clin Neurophysiol 60(2):130-134

Arendt-Nielsen L, Mills KR, Forster A (1989) Changes in muscle fiber conduction velocity, mean power frequency, and mean EMG voltage during prolonged submaximal contractions. Muscle Nerve 12(6):493-497

Arendt-Nielsen L, Gantchev N, Sinkjaer T (1992) The influence of muscle length on muscle fibre conduction velocity and development of muscle fatigue. Electroencephalogr Clin Neurophysiol 85(3):166-172

Bigland-Ritchie B, Donovan EF, Roussos CS (1981) Conduction velocity and EMG power spectrum changes in fatigue of sustained maximal efforts. J Appl Physiol 51(5):1300-1305

Broman H, Bilotto G, De Luca CJ (1985) Myoelectric signal conduction velocity and spectral parameters: influence of force and time. J Appl Physiol 58(5):1428-1437

Buchthal F, Dahl K, Rosenfalck P (1973) Rise time of the spike potential in fast and slowly contracting muscle of man. Acta Physiol Scand 87(2):261-269

Crenshaw AG, Karlsson S, Gerdle B, Friden J (1997) Differential responses in intramuscular pressure and EMG fatigue indicators during low-vs. high-level isometric contractions to fatigue. Acta Physiol Scand 160(4):353-361

Datta AK, Stephens JA (1990) Synchronization of motor unit activity during voluntary contraction in man. J Physiol 422:397-419

Eberstein A, Beattie B (1985) Simultaneous measurement of muscle conduction velocity and EMG power spectrum changes during fatigue. Muscle Nerve 8(9):768-773

Farina D, Fosci M, Merletti R (2002) Motor unit recruitment strategies investigated by surface EMG variables. J Appl Physiol 92(1):235-247

Farina D, Pozzo M, Merlo E, Bottin A, Merletti R (2004) Assessment of average muscle fiber conduction velocity from surface EMG signals during fatiguing dynamic contractions. IEEE Trans Biomed Eng 51(8):1383-1393

Gantchev N, Kossev A, Gydikov A, Gerasimenko Y (1992) Relation between the motor units recruitment threshold and their potentials propagation velocity at isometric activity. Electromyogr Clin Neurophysiol 32(4-5):221-228

Gazzoni M, Farina D, Merletti R (2001) Motor unit recruitment during constant low force and long duration muscle contractions investigated with surface electromyography. Acta Physiol Pharmacol Bulg 26(1-2):67-71

Gillies JD (1972) Motor unit discharge patterns during isometric contraction in man. J Physiol 223(1):36P-37P

Grimby L, Hannerz J (1968) Recruitment order of motor units on voluntary contraction: changes induced by proprioceptive afferent activity. J Neurol Neurosurg Psychiatr 31(6):565-573

Gydikov A, Christova L (1984) Effect of short interstimulus intervals on the electrically evoked potentials in human muscles. Electromyogr Clin Neurophysiol 24(1-2):137-153

Hagg G (1981) Electromyographic fatigue analysis based on the number of zero crossings. Pflugers Arch 391(1):78-80

Hagg GM, Suurkula J (1991) Zero crossing rate of electromyograms during occupational work and endurance tests as predictors for work related myalgia in the shoulder/neck region. Eur J Appl Physiol Occup Physiol 62(6):436-444

Henneman E, Somjen G, Carpenter DO (1965) Excitability and inhibitability of motoneurons of different sizes. J Neurophysiol 28(3):599-620

Hogrel JY, Duchene J, Marini JF (1998) Variability of some SEMG parameter estimates with electrode location. J Electromyogr Kinesiol 8(5):305-315

Houtman CJ, Stegeman DF, Van Dijk JP, Zwarts MJ (2003) Changes in muscle fiber conduction velocity indicate recruitment of distinct motor unit populations. J Appl Physiol 95(3):1045-1054

Hunter SK, Enoka RM (2003) Changes in muscle activation can prolong the endurance time of a submaximal isometric contraction in humans. J Appl Physiol 94(1):108-118

Hunter SK, Ryan DL, Ortega JD, Enoka RM (2002) Task differences with the same load torque alter the endurance time of submaximal fatiguing contractions in humans. J Neurophysiol 88(6):3087-3096

Hunter SK, Lepers R, MacGillis CJ, Enoka RM (2003) Activation among the elbow flexor muscles differs when maintaining arm position during a fatiguing contraction. J Appl Physiol 94(6):2439_ 2447

Inbar GF, Allin J, Paiss O, Kranz H (1986) Monitoring surface EMG spectral changes by the zero crossing rate. Med Biol Eng Comput 24(1):10-8

Komi PV, Tesch P (1979) EMG frequency spectrum, muscle structure, and fatigue during dynamic contractions in man. Eur J Appl Physiol Occup Physiol 42(1):41-50

Krogh-Lund C (1993) Myo-electric fatigue and force failure from submaximal static elbow flexion sustained to exhaustion. Eur J Appl Physiol Occup Physiol 67(5):389-401

Krogh-Lund C, Jorgensen K (1991) Changes in conduction velocity, median frequency, and root mean square-amplitude of the electromyogram during $25 \%$ maximal voluntary contraction of the triceps brachii muscle, to limit of endurance. Eur J Appl Physiol Occup Physiol 63(1):60-69

Krogh-Lund C, Jorgensen K (1992) Modification of myo-electric power spectrum in fatigue from $15 \%$ maximal voluntary contraction of human elbow flexor muscles, to limit of endurance: reflection of conduction velocity variation and/or centrally mediated mechanisms? Eur J Appl Physiol Occup Physiol 64(4):359-570

Kutner MH, Nachtsheim LC, Neter J, Li W (2005) Applied linear statistical models. McGraw-Hill/Irwin, New York, pp 1157-1162

Lange F, Van Weerden TW, Van Der Hoeven JH (2002) A new surface electromyography analysis method to determine spread of muscle fiber conduction velocities. J Appl Physiol 93(2):759-764

Lynn PA (1979) Direct on-line estimation of muscle fiber conduction velocity by surface electromyography. IEEE Trans Biomed Eng 26(10):564-571

MacGillis CJ, Semmler JG, Jacobi JM, Enoka RM (2003) Motor unit discharge differs with intensity and type of isometric contractions performed with the elbow flexor muscles. Med Sci Sport Exer $35: \mathrm{S} 280$ 
Masuda T, Sadoyama T (1986) The propagation of single motor unit action potentials detected by a surface electrode array. Electroencephalogr Clin Neurophysiol 63(6):590-598

Masuda T, Sadoyama T (1987) Skeletal muscles from which the propagation of motor unit action potentials is detectable with a surface electrode array. Electroencephalogr Clin Neurophysiol 67(5):421-427

Masuda T, Miyano H, Sadoyama T (1982) The measurement of muscle fiber conduction velocity using a gradient threshold zero-crossing method. IEEE Trans Biomed Eng 29(10):673-678

Miller RG, Giannini D, Milner-Brown HS, Layzer RB, Koretsky AP, Hooper D, Weiner MW (1987) Effects of fatiguing exercise on high-energy phosphates, force, and EMG: evidence for three phases of recovery. Muscle Nerve 10(9):810-821

Milner-Brown HS, Miller RG (1986) Muscle membrane excitation and impulse propagation velocity are reduced during muscle fatigue. Muscle Nerve 9(4):367-374

Milner-Brown HS, Stein RB, Yemm R (1973a). Changes in firing rate of human motor units during linearly changing voluntary contractions. J Physiol 230(2):371-390

Milner-Brown HS, Stein RB, Yemm R (1973b). The orderly recruitment of human motor units during voluntary isometric contractions. J Physiol 230(2):359-370

Mottram CJ, Jakobi JM, Semmler JG, Enoka RM (2005a) Motor-unit activity differs with load type during a fatiguing contraction. $J$ Neurophysiol 93(3):1381-1392

Mottram CJ, Christou EA, Meyer FG, Enoka RM (2005b) Frequency modulation of motor unit discharge has task-dependent effects on fluctuations in motor output. J Neurophysiol 94(4):2878-2887

Naeije M, Zorn H (1983) Estimation of the action potential conduction velocity in human skeletal muscle using the surface EMG crosscorrelation technique. Electromyogr Clin Neurophysiol 23(12):73-80

Nardone A, Romano C, Schieppati M (1989) Selective recruitment of high-threshold human motor units during voluntary isotonic lengthening of active muscles. J Physiol 409:451-471

Nishizono H, Kurata H, Miyashita M (1989) Muscle fiber conduction velocity related to stimulation rate. Electroencephalogr Clin Neurophysiol 72(6):529-534

Philippou A, Bogdanis GC, Nevill AM, Maridaki M (2004) Changes in the angle-force curve of human elbow flexors following eccentric and isometric exercise. Eur J Appl Physiol 93(1-2):237-244

Potvin JR (1997) Effects of muscle kinematics on surface EMG amplitude and frequency during fatiguing dynamic contractions. J Appl Physiol 82(1):144-151
Pozzo M, Merlo E, Farina D, Antonutto G, Merletti R, Di Prampero PE (2004) Muscle-fiber conduction velocity estimated from surface EMG signals during explosive dynamic contractions. Muscle Nerve 29(6):823-833

Radicheva N, Gerilovsky L, Gydikov A (1986) Effect of short interstimulus intervals on the intra- and extracellular action potentials of isolated frog muscle fibres. Acta Physiol Pharmacol Bulg 12(1):26-35

Rau G, Disselhorst-Klug C, Silny J (1997) Noninvasive approach to motor unit characterization: muscle structure, membrane dynamics and neuronal control. J Biomech 30(5):441-446

Rudroff T, Poston B, Shin IS, Bojsen-Moller J, Enoka RM (2005) Net excitation of the motor unit pool varies with load type during fatiguing contractions. Muscle Nerve 31(1):78-87

Rudroff T, Barry BK, Stone AL, Barry CJ, Enoka RM (2007) Accessory muscle activity contributes to the variation in time to task failure for different arm postures and loads. J Appl Physiol 102(3):1000-1006

Sadoyama T, Masuda T (1987) Changes of the average muscle fiber conduction velocity during a varying force contraction. Electroencephalogr Clin Neurophysiol 67(5):495-497

Sadoyama T, Masuda T, Miyano H (1985) Optimal conditions for the measurement of muscle fibre conduction velocity using surface electrode arrays. Med Biol Eng Comput 23(4):339-342

Semmler JG, Nordstrom MA (1999) A comparison of cross-correlation and surface EMG techniques used to quantify motor unit synchronization in humans. J Neurosci Methods 90(1):47-55

Sollie G, Hermens HJ, Boon KL, Wallinga-de Jonge W, Zilvold G (1985) The boundary conditions for measurements of the conduction velocity of the muscle fibers with surface EMG. Electromyogr Clin Neurophysiol 25:45-56

Stalberg E (1966) Propagation velocity in human muscle fibers in situ. Acta Physiol Scand Suppl 287:1-112

Van der Hoeven JH, Lange F (1994) Supernormal muscle fiber conduction velocity during intermittent isometric exercise in human muscle. J Appl Physiol 77(2):802-806

Van der Ploeg RJ, Oosterhuis HJ (1991) The "make/break test" as a diagnostic tool in functional weakness. J Neurol Neurosurg Psychiatry $54(3): 248-251$

Zwarts MJ, Arendt-Nielsen L (1988) The influence of force and circulation on average muscle fibre conduction velocity during local muscle fatigue. Eur J Appl Physiol Occup Physiol 58(3):278-283

Zwarts MJ, Van Weerden TW, Haenen HT (1987) Relationship between average muscle fibre conduction velocity and EMG power spectra during isometric contraction, recovery and applied ischemia. Eur J Appl Physiol Occup Physiol 56(2):212-216 